\title{
Interações sociais de crianças pré-escolares com Síndrome de Down durante atividades extracurriculares
}

\author{
Social interactions of preschool children with Down syndrome during extracurricular activities \\ Interacciones sociales de niños en edad preescolar con síndrome de Down durante actividades extracurriculares
}

\begin{abstract}
Renata Valdívia Lucisano', Luzia lara Pfeifer", Maria Paula Panuncio Pinto", Jair Lício Ferreira Santos"', Patrícia Páfaro Gomes Anhão"I

' Universidade de São Paulo, Faculdade de Medicina de Ribeirão Preto, Curso de Graduação em Terapia Ocupacional (Graduanda). Ribeirão Preto-SP, Brasil.

"Universidade de São Paulo, Faculdade de Medicina de Ribeirão Preto,

Departamento de Neurociências e Ciências do Comportamento. Ribeirão Preto-SP, Brasil.

I' Universidade de São Paulo, Faculdade de Medicina de Ribeirão Preto,

Departamento de Medicina Social, Programa de Pós-Graduação em Saúde na Comunidade. Ribeirão Preto-SP, Brasil.
\end{abstract}

Submissão: 16-02-2011 Aprovação: 09-02-2013

\section{RESUMO}

O objetivo desta pesquisa foi identificar o processo de interação social de crianças com Síndrome de Down (SD) durante atividades extracurriculares na rede regular de educação infantil do município de Ribeirão Preto. Participaram da pesquisa seis crianças na faixa etária de 3 a 6 anos. Foram realizadas duas filmagens de cada criança em situações de interação social durante atividades extracurriculares, e analisadas através de quinze comportamentos, distribuídos em duas categorias de habilidades sociais: interpessoais e de autoexpressão. Os resultados demonstraram que, quanto às habilidades interpessoais, houve maior ocorrência do comportamento "ocorre interação com outra criança". Já na categoria habilidades de autoexpressão, apenas os comportamentos "sorri" e "imita outras crianças" apresentam ocorrência significativa. Os comportamentos mais frequentes encontrados neste estudo permitem compreender que o ambiente escolar torna-se um facilitador na interação da criança com SD com outras crianças de desenvolvimento típico, possibilitando o desenvolvimento das habilidades sociais esperadas.

Descritores: Síndrome de Down; Interação Social; Criança.

\section{ABSTRACT}

The aim of this research was to identify the process of social interaction of children with Down Syndrome (DS) during extracurricular activities in the regular early childhood education in Ribeirão Preto-SP, Brazil. Six children aged 3-6 years participated in this study. There were two recordings of each child in situations of social interaction during extracurricular activities, and analyzed by 15 behaviors, divided into two categories of social skills: interpersonal and self-expression. The results demonstrate that, in the interpersonal skill category, the higher occurrence was the behavior "occurs interaction with other children". In the selfexpression skills category, only the behaviors "smiles" and "imitates other children" have significant occurrence. The behaviors more frequently identified in this study permit to understand that the school environment is a facilitator for the interaction of child with DS with the typical developmental children, allowing him/her to develop the expected social skills.

Key words: Down Syndrome; Interpersonal Relations; Child.

\section{RESUMEN}

El objetivo de esta investigación fue identificar el proceso de interacción social de los niños con Síndrome de Down (SD) en las actividades extraescolares en el jardín de niños regular en el municipio de Ribeirão Preto-SP, Brasil. Participaron en este estudio seis niños con edad entre 3 y 6 anos. Fueran realizadas dos grabaciones de cada niño en situaciones de interacción social durante las actividades extracurriculares, que se analizaron por 15 comportamientos, divididos en dos categorías de habilidades sociales: la interpersonal y la expresión personal. Los resultados demostraran que, en la categoría habilidades interpersonales, existe mayor incidencia de la conducta "está interactuando con otro niño". En la categoría habilidades de autoexpresión, sólo los comportamientos "sonrisas" y "imita a otros niños" tuvieran ocurrencia significativa. Los comportamientos más comunes que se encuentran en este estudio demuestran que el entorno escolar se convierte en un facilitador de la interacción del niño con síndrome de Down con otros niños con desarrollo normal, lo que permite el desarrollo de habilidades sociales esperadas. Palabras clave: Síndrome de Down; Relaciones Interpersonales; Niño. 


\section{INTRODUÇÃO}

A diversidade dos fatores biológicos, funções e realizações que existem em todos os seres humanos estão presentes, também, em crianças com Síndrome de Down (SD). O desenvolvimento mental e as habilidades intelectuais dessas crianças abrangem uma larga extensão entre o retardo mental e a inteligência próxima aos padrões considerados normais ${ }^{(1)}$.

Com relação aos marcos de desenvolvimento, as crianças com SD apresentam alguns atrasos em relação às não portadoras da síndrome. Há grande variabilidade no período de realizações do desenvolvimento em crianças com SD. Inúmeros fatores podem causar esse atraso, mas o principal deles é a ausência, no ambiente onde a criança vive, de condições para estímulos precoces e frequentes ${ }^{(1)}$.

Em relação às habilidades intelectuais, sabe-se que a deficiência mental é um quadro bastante frequente, havendo, entretanto, grande variabilidade das funções cognitivas para cada criança, já que a limitação de experiências físicas pode dificultar a exploração de objetos e do ambiente, interferindo no desenvolvimento cognitivo ${ }^{(2)}$. As crianças com deficiência mental apresentam semelhança no desenvolvimento cognitivo, percorrendo as mesmas etapas que uma criança de desenvolvimento típico, só que de forma mais lenta ${ }^{(3)}$, sendo que, com o passar dos anos, o processo de desenvolvimento das crianças com SD tende a se aproximar do que é tipicamente esperado, desde que sejam bem estimuladas ${ }^{(2)}$.

A criança com necessidades especiais de educação tem plenas condições para se desenvolver, principalmente quando Ihe são proporcionados meios que favoreçam esse desenvolvimento, ou seja, quando são feitas as adaptações e modificações necessárias ${ }^{(1)}$.

Os resultados apontados pelos autores demonstram a necessidade de se entender e promover a inclusão não meramente na esfera física, mas principalmente na esfera social, possibilitando uma participação mais ativa das pessoas com SD no processo de aprendizagem e interações sociais, em que haja realmente uma prática inclusiva e transformadora no ambiente escolar ${ }^{(1)}$.

Destacam-se aspectos que, uma vez garantidos, podem maximizar a possibilidade de sucesso no processo de inclusão: o planejamento desde a localização do aluno com necessidades especiais na sala de aula, até os cuidados com a qualificação dos professores para lidar com a diversidade de exigências, os cuidados na proposição de normas e procedimentos que regulam a organização de ensino, entre outros ${ }^{(4)}$.

A Educação Infantil tem por objetivo permitir que a criança desenvolva uma imagem positiva de si, atuando de forma cada vez mais independente, com confiança em suas capacidades e percepção de suas limitações; descobrir e conhecer progressivamente seu próprio corpo, suas potencialidades e seus limites, desenvolvendo e valorizando hábitos de cuidado com a própria saúde e bem-estar; estabelecer vínculos afetivos e de troca com adultos e outras crianças, fortalecendo sua auto-estima e ampliando gradativamente suas possibilidades de comunicação e interação social; estabelecer e ampliar cada vez mais as relações sociais, aprendendo aos poucos a articular seus interesses e pontos de vista com os demais, respeitando a diversidade e desenvolvendo atitudes de ajuda e colaboração; observar e explorar o ambiente com atitude de curiosidade, percebendo-se cada vez mais como integrante, dependente e agente transformador do meio ambiente e valorizando atitudes que contribuam para sua conservação; brincar, expressando emoções, sentimentos, pensamentos, desejos e necessidades; utilizar as diferentes linguagens (corporal, musical, plástica, oral e escrita) ajustadas às diferentes intenções e situações de comunicação, de forma a compreender e ser compreendido, expressar suas idéias, sentimentos, necessidades e desejos e avançar no seu processo de construção de significados, enriquecendo cada vez mais sua capacidade expressiva; conhecer algumas manifestações culturais, demonstrando atitudes de interesse, respeito e participação frente a elas e valorizando a diversidade ${ }^{(5)}$.

Logo, o processo de interação social é uma das mais importantes tarefas do desenvolvimento inicial da criança, uma vez que se caracteriza pela ampliação e refinamento do repertório de comportamentos sociais e, simultaneamente, pela compreensão gradual de valores e de normas que regulam o funcionamento da vida em sociedade(6).

As interações estabelecidas no micro sistema família são as que trazem implicações mais significativas para o desenvolvimento da criança, embora outros sistemas sociais como a escola, o local de trabalho dos pais, o templo religioso, e os clubes sociais que a família frequenta, contribuem também para o seu desenvolvimento. Infelizmente, há uma escassez de trabalhos que focalizam este tema, em se tratando de crianças que apresentam algum tipo de deficiência, particularmente no Brasil ${ }^{(7)}$.

Independente da cultura, período histórico, localização geográfica ou composição interna, a família é o primeiro grupo social ao qual o ser humano pertence. Dessa forma, o papel da família é fundamental e determinante no desenvolvimento da personalidade, atitude e modo de agir do indivíduo, mesmo em idade adulta. É através das relações estabelecidas com os integrantes de sua família - mãe, pai e demais membros - e das reações destes ao seu comportamento, que a criança tem seu primeiro contato com o mundo e aprende a desenvolver os papéis e atitudes essenciais ao seu processo de socialização ${ }^{(8)}$.

Na família, mais do que em qualquer outro grupo social, as fronteiras individuais são fluidas, e há uma constante troca de afetos, influências mútuas, expectativas e cobranças, conscientes ou não. Mesmo quando há conflitos e divergências internas, a família se comporta como uma unidade e, tudo o que acontece com um dos membros, afeta, diretamente, todos os demais ${ }^{(9)}$.

A inclusão social de pessoas com deficiências está diretamente ligada à dinâmica das relações vivenciadas na família e, sobretudo, à sua inclusão e integração na vida familiar cotidiana. O nível de inclusão que ela pode vir a desenvolver depende, em grande medida, da disponibilidade da sua família em Ihe permitir participar de diferentes ambientes e relações sociais, apesar das barreiras físicas e sociais existentes. $\mathrm{E}$, quanto maior a participação desse indivíduo em contextos sociais distintos, maior será possibilidade de assumir um novo papel no quadro familiar ${ }^{(9)}$. 
A convivência em um ambiente solicitador, que promova uma diversidade de estímulos e diferentes possibilidades de descobertas, permitirá a reorganização e a plasticidade cerebral do indivíduo ${ }^{(10)}$. É inegável que os adultos são extremamente influentes na vida de uma criança em relação ao desenvolvimento cognitivo e social, entretanto, as crianças também podem aprender com seus pares ${ }^{(11)}$. Desta maneira, o ambiente escolar passa a ser um importante espaço para aprendizagem e desenvolvimento de habilidades sociais.

O ingresso na escola é o primeiro contato da criança com o mundo, sua primeira experiência fora do ambiente familiar. É um grande passo e também um desafio para todos que participam dessa nova etapa. Para a compreensão do desenvolvimento da criança é necessário que ela seja observada não apenas em sua individualidade, como também em suas relações sociais. É importante observar de que forma a criança se expressa no grupo para que se possa compreendê-la. A escola poderá auxiliar no desenvolvimento de sua identidade individual, e através da relação com o outro irá favorecer de forma determinante suas relações sociais futuras ${ }^{(12)}$.

A inclusão escolar é uma prática cada vez mais frequente e a maneira como ela acontece está intimamente relacionada à cultura local e sua política ${ }^{(13)}$ e por isso pode acontecer de maneira variada nas cidades, regiões e países ${ }^{(14)}$.

Estudos mostram que a inclusão propicia às crianças com deficiência ganhos em vários aspectos, não apenas quanto aos benefícios acadêmicos, mas também referente às habilidades relativas à fala e ao comportamento social ${ }^{(14)}$.

Ao se fazer uma interligação entre o processo de interação social e a inclusão escolar de crianças com SD, é possível considerar que, ao entrarem na escola, a relação interpessoal com os colegas abre-lhes uma maior variabilidade de modelos e demandas para a aquisição de novas habilidades sociais. O desempenho social e a qualidade dos relacionamentos na escola têm como base os recursos comportamentais previamente adquiridos pela criança no contexto familiar. Com o advento das atuais políticas de inclusão, entende-se que o desenvolvimento interpessoal (particularmente nas habilidades de resolução de problemas, autocontrole e comportamentos pró-sociais) é componente indispensável desse processo. Essa posição é compatível com a adotada por vários pesquisadores que defendem, como objetivos principais da inclusão, a meIhoria da qualidade do relacionamento entre colegas, a promoção de atitudes de compreensão e aceitação das diferenças por parte de colegas e de professores ${ }^{(6)}$.

As habilidades sociais são aprendidas e as demandas para a sua aquisição de desempenho variam em função do estágio do indivíduo, como sendo resultado de contingências ambientais, às quais ele é exposto ${ }^{(15)}$. Desta forma, uma criança da Educação Infantil não demonstra as mesmas habilidades sociais de outra que cursa o Ensino Fundamental, assim como as habilidades que um adolescente demonstra não são as mesmas esperadas de um adulto ou pessoa idosa(16).

Em estudo sobre inclusão e interação social de crianças com SD na educação infantil, concluíram que nos comportamentos observados e de acordo com a faixa etária estudada, o grupo de crianças com SD abordado, não apresentou características de interação social muito diferente das crianças com desenvolvimento típico, reforçando a importância do processo de inclusão escolar desta população(16).

O presente estudo busca, por meio de uma abordagem analítica, descritiva e observacional, levantar alguns aspectos significativos das habilidades sociais de crianças com SD, quando inseridas em ambientes inclusivos através do processo de interação durante atividades extracurriculares com outras crianças com desenvolvimento típico, quando inseridas em escolas de educação infantil.

\section{METODOLOGIA}

Esta é uma pesquisa de estudo de casos múltiplos, não experimental, transversal, de caráter qualitativo e quantitativo, descritivo. O projeto foi aprovado pelo Comitê de Ética da Faculdade de Medicina de Ribeirão Preto da USP, em 11 de fevereiro de 2008, sob o Protocolo no 9296/2008, seguindo as normas estabelecidas pela Resolução 196/96 do Conselho Nacional de Saúde.

Para participar da presente pesquisa as crianças precisavam ter o diagnóstico de Síndrome de Down, estarem inseridas em Escolas Municipais de Educação Infantil (EMEI) de Ribeirão Preto e serem egressas do Programa de Estimulação Precoce da APAE do mesmo município. Foram localizadas seis crianças, com idade entre 5 e 6 anos, sendo duas meninas e quatro meninos, as quais compuseram a amostra deste estudo. O número reduzido de participantes não prejudicou o alcance dos objetivos, por se tratar de um estudo de caráter descritivo observacional, tendo como enfoque a qualidade de interações sociais que as crianças apresentam.

As crianças foram observadas durante as atividades extracurriculares, as quais aconteciam em ambientes externos tais como quadra, parque de areia, campo de futebol, sala de brinquedos, os quais eram semelhantes em todas as escolas, por se tratarem de EMEIs, contendo brinquedos grandes em ferro (escorrega, gira-gira, túnel, gangorra, casinha suspensa), brinquedos de areia (baldinho, peneira, pá). Havia também outros objetos de acordo com as atividades propostas no dia da coleta, tais como bola, bonecas, panelinhas, carrinhos e brinquedos de encaixe.

O registro do comportamento das crianças foi feito com câmera filmadora digital, em duas sessões de quinze minutos, totalizando meia hora de filmagem para cada criança participante do estudo. Cada sessão de filmagem foi realizada em dias diferentes, a fim de realizar uma abordagem mais ampla de cada criança. Situações de curiosidade infantil, que ocorreram durante as filmagens, foram solucionadas com respostas simples e curtas, a fim de não influenciarem a dinâmica do ambiente de interação social.

A filmadora foi focalizada na criança participante do estudo, e a pesquisadora procurou não estabelecer nenhum tipo de interação durante as filmagens, sendo que o início da filmagem foi determinado pelo início da atividade proposta.

Em uma ficha de registro, contendo cada um dos comportamentos analisados, foi assinalado o minuto em que o comportamento foi observado. As duas sessões de filmagens 
foram contabilizadas conjuntamente para obtenção de uma média de comportamentos apresentados pelos participantes.

Os dados coletados na filmagem foram analisados quali-quantitativamente a partir dos quinze comportamentos distribuídos em duas categorias de habilidades sociais (interpessoais e de autoexpressão) propostas por Anhão e colaboradores ${ }^{(17)}$, apresentadas no quadro 1 a seguir. O Teste de Friedman ${ }^{(18)}$ foi empregado para verificar a ocorrência de diferenças nas pontuações das atividades relativas às habilidades interpessoais e às de autoexpressão, ao nível de significância de $5 \%$.

\begin{tabular}{ll}
\hline CATEGORIAS & \multicolumn{1}{c}{ COMPORTAMENTOS } \\
\hline & Ocorre interação com outra criança \\
& Ocorre interação com adulto (educador) \\
& $\begin{array}{l}\text { Ocorre interação com objetos } \\
\text { (brinquedos, material didático) }\end{array}$ \\
& Disputa a atenção da educadora com outra pessoa \\
Habilidades & Ocorrem brigas ou agressões \\
& Apresenta autodefesa \\
& Estabelece contato inicial com outras crianças \\
& Brinca junto, mas com objetos diferentes \\
& Brinca junto com o mesmo tipo de objeto \\
& Chora \\
& Sorri \\
& Fica sozinho \\
Habilidades de \\
autoexpressão
\end{tabular}

Imita outras crianças

Imita a educadora

Quadro 1 - Apresentação dos quinze comportamentos, distribuídos nas categorias de habilidades sociais.

\section{RESULTADOS}

Os resultados demonstram que, quanto às habilidades interpessoais houve uma maior ocorrência do comportamento "ocorre interação com outra criança", com média de 28.3 na frequência de comportamentos apresentados, na somatória das duas filmagens de cada criança.

Em seguida aparece a categoria "brinca junto com o mesmo tipo de objeto" com média de 21 na frequência de comportamentos apresentados pelas crianças do estudo. O comportamento "ocorre interação com objetos" pode ser visualizado logo depois, com média de 15.5 na frequência dos comportamentos.

O comportamento "ocorre interação com a educadora" ocorre com uma frequência mais baixa sendo a média de 7.5. Já o comportamento "estabelece contato inicial com outra pessoa", foi observado com uma frequência média de 6.6.
Os comportamentos "disputa a atenção da educadora com outra pessoa", "ocorrem brigas ou agressões" e "tem autodefesa" foram pouco presentes com frequência média menor que 1 .

Os resultados acima citados, referentes às habilidades interpessoais, podem ser vistos mais detalhadamente na figura 1:

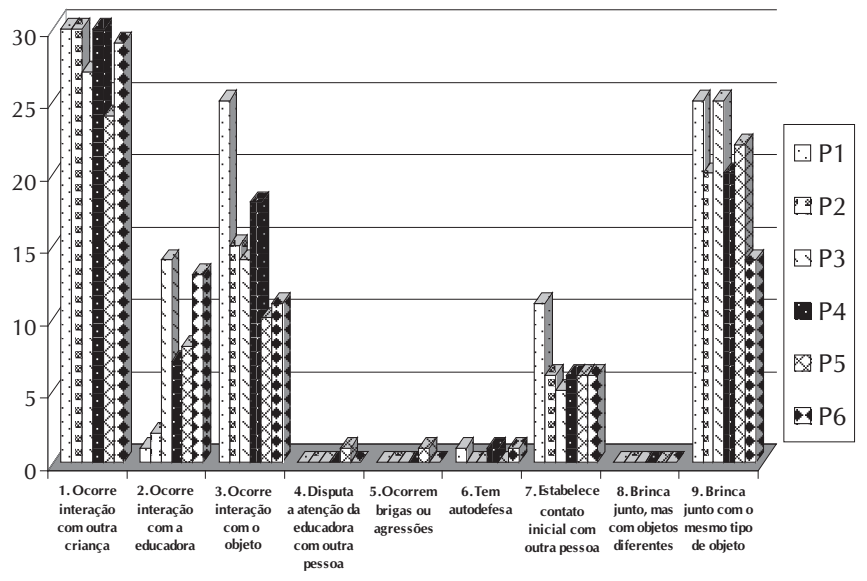

Figura 1 - Frequência das habilidades interpessoais de cada participante

Dentre os comportamentos da categoria habilidades de autoexpressão, apenas o comportamento "sorri" e "imita outras crianças" apresentou uma ocorrência significativa com média de 5.16 e 3 respectivamente na frequência de comportamentos apresentados, na somatória das duas filmagens de cada criança. O comportamento "fica sozinho" apresentou média de 2.16 na frequência dos comportamentos e os outros comportamentos apresentaram uma frequência média abaixo de 1, como pode ser observado na figura 2 a seguir:

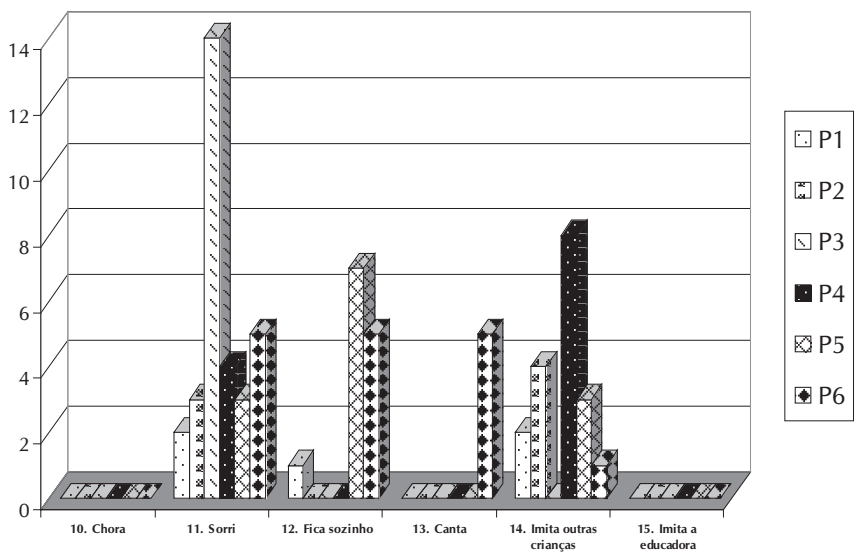

Figura 2 - Frequência das habilidades de autoexpressão de cada participante

Em relação às diferenças entre cada um dos nove comportamentos que compõem as habilidades interpessoais foram calculados a média, mediana, desvio padrão e os valores máximo e mínimo, conforme pode ser visualizado na tabela 1 a seguir: 
Tabela 1 - Dados descritivos das habilidades interpessoais:

\begin{tabular}{|c|c|c|c|c|c|c|c|c|c|}
\hline & $\begin{array}{l}\text { Interação } \\
\text { c/ outra } \\
\text { criança }\end{array}$ & $\begin{array}{c}\text { Interação } \\
\text { c/ adulto }\end{array}$ & $\begin{array}{c}\text { Interação } \\
\text { c/ } \\
\text { objetos }\end{array}$ & $\begin{array}{c}\text { Disputa a } \\
\text { atenção do } \\
\text { educador c/ } \\
\text { outra pessoa }\end{array}$ & $\begin{array}{l}\text { Brigas ou } \\
\text { agressões }\end{array}$ & $\begin{array}{c}\text { Auto } \\
\text {-defesa }\end{array}$ & $\begin{array}{c}\text { Estabelece } \\
\text { contato inicial } \\
\text { c/ outras } \\
\text { crianças }\end{array}$ & $\begin{array}{c}\text { Brinca } \\
\text { junto, mas } \\
\text { c/ objetos } \\
\text { diferentes }\end{array}$ & $\begin{array}{c}\text { Brinca junto } \\
\text { c/ o mesmo } \\
\text { tipo de } \\
\text { objeto }\end{array}$ \\
\hline Média & 24.5 & 10.2 & 16.0 & 1.7 & 0.2 & 0.7 & 5.7 & 1.0 & 17.3 \\
\hline Mediana & 28.5 & 7.5 & 14.5 & 0.0 & 0.0 & 1.0 & 6.0 & 0.0 & 20.0 \\
\hline $\begin{array}{l}\text { Desvio } \\
\text { Padrão }\end{array}$ & 9.4 & 10.3 & 5.0 & 4.1 & 0.4 & 0.5 & 3.5 & 2.4 & 9.4 \\
\hline $\begin{array}{l}\text { Valor } \\
\text { Máximo }\end{array}$ & 30.0 & 29.0 & 25.0 & 10.0 & 1.0 & 1.0 & 11.0 & 6.0 & 25.0 \\
\hline $\begin{array}{l}\text { Valor } \\
\text { Mínimo }\end{array}$ & 6.0 & 1.0 & 11.0 & 0.0 & 0.0 & 0.0 & 0.0 & 0.0 & 0.0 \\
\hline
\end{tabular}

Realizado o Teste de Friedman, os resultados encontrados demonstraram haver diferenças significativas entre os comportamentos que compõem a categoria das habilidades interpessoais com $p<0.01$.

Em relação às diferenças entre cada um dos seis comportamentos que compõem a categoria das habilidades de autoexpressão foram calculados a média, mediana, desvio padrão e os valores máximo e mínimo, conforme pode ser visualizado na tabela 2 a seguir.

Tabela 2 - Dados descritivos das Habilidades de Autoexpressão:

\begin{tabular}{lcccccc}
\hline & Chora & Sorri & $\begin{array}{c}\text { Fica } \\
\text { sozinho }\end{array}$ & Canta & $\begin{array}{c}\text { Imita } \\
\text { outras } \\
\text { crianças }\end{array}$ & $\begin{array}{c}\text { Imita a } \\
\text { educadora }\end{array}$ \\
\hline Média & 3.7 & 4.7 & 1.5 & 2.0 & 2.5 & 0.5 \\
Mediana & 0.0 & 3.5 & 0.5 & 0.0 & 1.5 & 0.0 \\
Desvio Padrão & 9.0 & 4.9 & 2.1 & 3.2 & 3.1 & 1.2 \\
Valor Máximo & 22.0 & 14.0 & 5.0 & 7.0 & 8.0 & 3.0 \\
Valor Mínimo & 0.0 & 0.0 & 0.0 & 0.0 & 0.0 & 0.0 \\
\hline
\end{tabular}

Através do Teste de Friedman, os resultados encontrados demonstram que há diferenças significativas entre os comportamentos que compõem a categoria das habilidades de autoexpressão com $p=0.02$.

\section{DISCUSSÃO}

Os resultados são discutidos a partir dos comportamentos mais observados em cada uma das duas categorias de análises, habilidades interpessoais e de autoexpressão.

Habilidades sociais são comportamentos aprendidos e aceitáveis socialmente permitindo que o sujeito apresente uma interação efetiva com seus pares evitando ou fugindo de comportamentos não aceitáveis que resultem em interações sociais negativas ${ }^{(6)}$. As habilidades sociais de crianças e adolescentes facilitam a iniciação e manutenção de relações sociais positivas, contribuindo com a aceitação de colegas e resultando em ajustamento escolar satisfatório ${ }^{(6)}$.

A inserção de alunos com necessidades especiais no sistema regular de ensino deve ser planejada de maneira a reunir em uma mesma turma, alunos com idades similares, que participem conjuntamente de todas as atividades escolares, ainda que tenham objetivos de aprendizagem com diferentes graus de complexidade. A inclusão de pessoas com necessidades especiais no sistema regular de ensino é um processo complexo que requer o envolvimento e a participação de todos os integrantes das organizações escolares. A produção de conhecimento sobre as interações entre alunos com SD, seus colegas de turma e professores no sistema regular de ensino, constitui uma necessidade científica e social, uma vez que oferece subsídios para a caracterização e aperfeiçoamento de processos de inclusão escolar ${ }^{(4)}$.

\section{Habilidades Interpessoais}

Observa-se que os comportamentos "Ocorre interação com outra criança" e "Ocorre interação com objeto" estão entre os três comportamentos que tiveram maior frequência. A comunicação verbal e não verbal, ou seja, interação com outros ou com objetos, são os componentes básicos ao desenvolvimento social, e a capacidade de utilizá-los de forma coerente e complementar é ingrediente fundamental para um desempenho socialmente competente ${ }^{(6)}$.

Quando a criança passa a frequentar outros microssistemas além do familiar, ampliam-se a quantidade e a diversidade de interlocutores, assim como as oportunidades para aplicar e aperfeiçoar seu repertório social, o que influi decisivamente na sua aquisição e desempenho das habilidades sociais. Considerando-se a relação com colegas, ao entrar na escola abre-se, para a criança, uma maior variabilidade de modelos e demandas para a aquisição de novas habilidades sociais ${ }^{(6)}$.

Uma vez que as crianças tomam para si as normas do grupo, é interessante estudar a presença de alunos com deficiência no ambiente regular de ensino, assim como as interações sociais que ocorrem naturalmente entre alunos com deficiência e os demais, focalizando o papel do outro como mediador 
de sua interação com a sociedade ${ }^{(19)}$.

Dessa forma, é possível inferir que as crianças do estudo quando inseridas nas atividades extracurriculares do ambiente escolar aprendem com seus pares, desenvolvendo as habilidades sociais, e desempenhando-as durante este convívio.

Os comportamentos "brinca junto com o mesmo tipo de objeto" e "brinca junto, mas com objetos diferentes" estão relacionados, e dizem respeito aos momentos de brincadeira, e de como os objetos são utilizados durante esta brincadeira. Porém, é possível perceber que apenas o comportamento "brinca com o mesmo tipo de objeto" foi observado com uma frequência bastante expressiva. Essa situação pode sugerir que as crianças, quando inseridas em um mesmo ambiente tendem a brincar com o mesmo tipo de objeto, ou elaboram suas brincadeiras de maneira a atingir os mesmos objetivos, sendo necessária, para tanto, a utilização do mesmo tipo de objeto ${ }^{(17)}$.

Os comportamentos "disputa a atenção da educadora", "ocorrem brigas ou agressões" e "apresentam autodefesa", demonstram como os participantes reagem a situações conflitantes, e ocorreu com frequência bastante baixa, o que demonstra que as crianças com SD estavam bem adaptadas ao ambiente escolar e na interação com seus parceiros de classe.

Estudos mostram que a inclusão propicia às crianças com deficiência ganhos em vários aspectos, como, por exemplo, nas habilidades relativas à fala, ao comportamento social e aos benefícios acadêmicos ${ }^{(14)}$. Tal fato pode estar relacionado à diversidade de estímulos oferecidos no ambiente escolar, pois, um ambiente solicitador, que promova diferentes possibilidades de descobertas, permitirá a reorganização e a plasticidade cerebral do indivíduo ${ }^{(10)}$.

Já o comportamento "ocorre interação com a educadora" apresentou uma frequência baixa, o que pode sugerir que, neste ambiente, as crianças interagem mais com seus pares, por serem realizadas atividades não estruturadas pela professora e, assim, as crianças ficam mais livres para brincar. É importante perceber que através da inclusão escolar as crianças passam a conviver com seus pares ampliando seu universo de interação social e apresentando um comportamento semeIhante ao de crianças de desenvolvimento típico.

Com relação ao comportamento "estabelece contato inicial com outra pessoa", quando inseridos no ambiente externo foi verificada uma frequência relativamente baixa. Tal informação pode sugerir que as crianças com SD apresentam maior dificuldade em iniciar o contato social, talvez como consequência da falta de oportunidades de convivência em ambientes sociais externos aos familiares. Há uma classificação sobre este tipo de comportamento como habilidades sociais de comunicação, em seu estudo sobre o repertório social de indivíduos adolescentes com $\mathrm{SD}^{(15)}$. O mesmo autor verificou que nas situações estruturadas investigadas, a maioria dos participantes, com SD, apresentou um déficit de respostas assertivas de enfrentamento, em seu repertório comportamental ${ }^{(15)}$.

\section{Habilidades de autoexpressão}

As interações sociais são entendidas como educativas à medida que representam condições para a aquisição de conceitos, habilidades e estratégias cognitivas que afetam o desenvolvimento social e a aprendizagem ${ }^{(6)}$. As interações sociais satisfatórias da criança, com colegas e professores, requerem um repertório adequado de habilidades sociais, ou seja, de diferentes classes de comportamentos sociais para lidar de maneira adequada com as demandas das situações interpessoais $^{(6)}$. As dificuldades em habilidades sociais, definidas como déficits de aquisição ou de desempenho adequado às demandas da situação e da cultura, interferem, portanto, na qualidade dessas relações ${ }^{(20)}$.

Dentre as habilidades de autoexpressão, o comportamento "sorri" apresentou a maior ocorrência, o que sugere que as crianças com SD sentem-se bem quando estão interagindo com seus pares durante as atividades extracurriculares.

Já os comportamentos "chora" e "canta" abordam as questões de expressão das emoções, assim como o comportamento "sorri", porém os dois primeiros apareceram pouquíssimas vezes. Isso não significa que estes comportamentos não ocorram de fato com maior frequência. Pode-se supor que, durante a realização das filmagens tais comportamentos ocorreram de forma pouco expressiva.

O comportamento "fica sozinho" também apresentou uma frequência baixa de ocorrência, o que pode indicar que as crianças com SD apresentam potencial para interagir com seus pares, quando isso lhes é oportunizado. Pressupõe-se que a proposta de inclusão escolar de crianças com necessidades educativas especiais procura evitar os efeitos deletérios do isolamento social dessas crianças, criando oportunidades para a interação entre as crianças, inclusive como forma de diminuir o preconceito ${ }^{(19)}$.

Em relação aos comportamentos "imita outra(s) criança(s)" e "imita a educadora", o primeiro apresentou uma frequência muito mais significativa. Isso não quer dizer que a educadora não apresente papel fundamental no processo de interação social e inclusão, mas que crianças, nesta faixa etária, solicitam mais a interação com outras crianças as quais apresentem os mesmos interesses. A educadora esteve presente em todos os momentos possibilitando que as crianças pudessem desenvolver suas habilidades sociais ${ }^{(17)}$.

Ainda segundo esses mesmos autores, essas informações podem sugerir que as crianças com SD têm uma maior tendência a imitar os comportamentos apresentados por outras crianças, e não de buscar o seu "modelo" de atuação junto aos educadores. Diante disso, destaca-se a importância do ambiente escolar no processo de inclusão, já que este possibilita um maior contato da criança com SD, com outras crianças de desenvolvimento típico, na mesma faixa etária, o que não ocorre em ambientes protegidos de ensino especial ou mesmo em setting terapêutico ${ }^{(17)}$.

\section{CONCLUSÃO}

Os resultados desta pesquisa mostram que o processo de desenvolvimento das interações sociais das crianças com SD acontece de forma bastante próxima de seus pares, diferindo apenas no ritmo e na forma de como as crianças com SD buscam e sustentam esta relação. Assim, é importante que 
ocorram mudanças práticas na educação, no intuito de buscar a inclusão real desses alunos no ambiente de ensino regular, transformando em ações que sejam benéficas para o amadurecimento e desenvolvimento não só desses alunos, mas também daquelas crianças com desenvolvimento típico.

Os comportamentos classificados como de habilidades interpessoais, encontrados com maior frequência, permitem compreender que o ambiente escolar torna-se um facilitador na promoção de um maior contato da criança com SD com outras crianças da mesma faixa etária, possibilitando o desenvolvimento das habilidades sociais das crianças do estudo.

Portanto, é possível reafirmar a importância de crianças com SD estarem inseridas na rede regular de ensino, favorecendo assim o convívio com educadores e seus pares, facilitando a aquisição de habilidades sociais e desempenho de comportamentos necessários na vida em sociedade.

\section{REFERÊNCIAS}

1. Reis Filho AD, Schuller JAP. A capoeira como instrumento pedagógico no aprimoramento da coordenação motora de pessoas com Síndrome de Down. Pensar Prátic 2010;13(2):1-21.

2. Bonomo LMM, Rossetti CB. Aspectos percepto-motores e cognitivos do desenvolvimento de crianças com Síndrome de Down. Rev Bras Crescimento Desenvolv Hum 2010;20(3):723-734.

3. Ciciliato MN, Zilotti DC, Mandrá PP. Caracterização das habilidades simbólicas de crianças com Síndrome de Down. Rev Soc Bras Fonoaudiol 2010;15(3):408-14.

4. Teixeira FC, Kubo OM. Características das interações entre alunos com Síndrome de Down e seus colegas de turma no sistema regular de ensino. Rev Bras Ed Esp 2008; 14(1):75-92.

5. Brasil. Ministério da Educação e do Desporto [homepage na internet]. Secretaria de Educação Fundamental. Referencial curricular nacional para a educação infantil. Ministério da Educação e do Deporto, Secretária da Educação Fundamental. Brasília: MEC/SEF; 1998 [acesso em 05 jan 2011]. Disponível em: http://portal.mec.gov.br/seb/ arquivos/pdf/rcnei vol1.pdf.

6. Del Prette ZAP, Del Prette A. Psicologia das habilidades sociais na infância: Teoria e Prática. 4. ed. Petrópolis: Vozes; 2009.

7. Dessen MA, Silva NLP. Deficiência mental e família: Uma análise da produção científica. Paidéia(Ribeirão Preto) 2000;10(19):12-23.

8. Ferraz CRA, Araújo MV, Carreiro LRR. Inclusão de crianças com Síndrome de Down e paralisia cerebral no ensino fundamental I: comparação dos relatos de mães e professores. Rev Bras Educ Espec 2010;16(3):397-414.

9. Glat R, Pletsch MD. Orientação familiar como estratégia facilitadora do desenvolvimento e inclusão de pessoas com necessidades especiais. Rev Bras Educ Espec 2004;(24):1-5.
10. Silva MFMC, Kleinhans ACS. Processos cognitivos e plasticidade cerebral na síndrome de Down. Rev Bras Educ Espec 2006;12(1):123-138.

11. Flynn E, Whiten A. Studying children's social learning experimentally "in the wild". Learn Behav 2010;38(3):284-296.

12. Pacheco WS, Oliveira MS. Aprendizagem e desenvolvimento da criança com Síndrome de Down: representações sociais de mães e professoras. Cien Cogn 2011;16(3):2-14.

13. Luiz FMR, De Bortoli PS, Floria-Santos M, Nascimento LC. A inclusão da criança com síndrome de Down na rede regular de ensino: desafios e possibilidades. Rev Bras Educ Espec 2008;14(3):497-508.

14. Buckley S, Bird G, Sacks B, Archer T. A comparison of mainstream and special education for teenagers with Down syndrome: implications for parents and teachers. Downs Syndr Res Pract. 2006;9(3):54-67.

15. Angélico AP, Del Prette A. Avaliação do Repertório de Habilidades Sociais de Adolescentes com Síndrome de Down. Psicol Reflex Crít 2011;24(2):207-217.

16. Soresi S, Nota L. A social skill training for persons with Down's Syndrome. Eur Psychol 2000;5(1):33-43.

17. Anhao PPG, Pfeifer LI, Santos JL. Interação social de crianças com Síndrome de Down na educação infantil. Rev Bras Educ Espec 2010;16(1):31-46.

18. Siegel $\mathrm{S}$, Castellan Jr NJ. Estatística não-paramétrica para ciências do comportamento. 2.ed. Porto Alegre: Penso; 2006.

19. Batista MW, Enumo SRF. Inclusão escolar e deficiência mental: análise da interação social entre companheiros. Estud Psicol 2004;9(1):101-11.

20. Molina RCM, Del Prette ZAP. Funcionalidade da relação entre habilidades sociais e dificuldades de aprendizagem. Psico-USF 2006;11(1):53-63. 